\title{
Evaluating the submission of digital images as a method of surveillance for Ixodes scapularis ticks
}

\author{
J. K. KOFFI ${ }^{1}+\dagger$, J. SAVAGE ${ }^{2} \dagger$, K. THIVIERGE ${ }^{3} \dagger$, L. R. LINDSAY ${ }^{4}$, C. BOUCHARD ${ }^{5}$, \\ Y. PELCAT ${ }^{5}$ and N.H. OGDEN ${ }^{5}$ \\ ${ }^{1}$ Zoonoses Division, Centre for Foodborne Environmental and Zoonotics Infectious Diseases, Public Health Agency of \\ Canada, 3200 Sicotte Street, PO 5000, Saint-Hyacinthe, Québec, Canada F2S 7C6 \\ ${ }^{2}$ Biological Sciences, Bishop’s University, 2600 College Street, Sherbrooke, Québec, Canada F1M $1 Z 7$ \\ ${ }^{3}$ Laboratoire de santé publique du Québec, Institut National de Santé Publique du Québec 20045, chemin Sainte-Marie, \\ Sainte-Anne-de-Bellevue, Québec, Canada H9X 3R5 \\ ${ }^{4}$ Zoonotic Diseases and Special Pathogens, National Microbiology Laboratory, Public Health Agency of Canada, 1015 \\ Arlington Street, Winnipeg, Manitoba, Canada R3E 3R2 \\ ${ }^{5}$ Public Health Risk Sciences Division, National Microbiology Laboratory, Public Health Agency of Canada, 3200 Sicotte \\ Street, PO 5000, Saint-Hyacinthe, Québec, Canada Y2S $7 C 6$
}

(Received 15 November 2016; revised 14 December 2016; accepted 3 Fanuary 2017; first published online 27 March 2017)

\section{SUMMARY}

Widespread access to the internet is offering new possibilities for data collection in surveillance. We explore, in this study, the possibility of using an electronic tool to monitor occurrence of the tick vector of Lyme disease, Ixodes scapularis. The study aimed to compare the capacity for ticks to be identified in web-based submissions of digital images/photographs, to the traditional specimen-based identification method used by the provincial public health laboratory in Quebec, Canada. Forty-one veterinary clinics participated in the study by submitting digital images of ticks collected from pets via a website for image-based identification by an entomologist. The tick specimens were then sent to the provincial public health laboratory to be identified by the 'gold standard' method using a microscope. Of the images submitted online, $74 \cdot 3 \%$ (284/382) were considered of high-enough quality to allow identification. The laboratory identified 382 tick specimens from seven different species, with $I$. scapularis representing $76 \%$ of the total submissions. Of the 284 ticks suitable for image-based species identification, $276(97 \cdot 2 \%)$ were correctly identified (Kappa statistic of $0 \cdot 92, Z=15 \cdot 46, P<0 \cdot 001$ ). This study demonstrates that image-based tick identification may be an accurate and useful method of detecting ticks for surveillance when images are of suitable quality.

Key words: Lyme disease, tick-borne diseases, surveillance, electronic platform, Ixodes scapularis, Ixodes cookei, Dermacentor spp.

\section{INTRODUCTION}

Tick-borne diseases (TBDs) are the most prevalent vector-borne diseases in North America and in Europe and include Lyme borreliosis, Anaplasmosis, Babesiosis, rickettsial infections and tick-borne relapsing fevers and encephalitis. One of the most studied TBD is Lyme disease (LD), which is transmitted by several hard tick species in the genus Ixodes. Ixodes scapularis and Ixodes pacificus are the main vectors in North America, while Ixodes persulcatus and Ixodes ricinus are the main vectors in Eurasia. Increasing temperatures associated with global climate change are likely driving northward expansion of $I$. scapularis in North America (Brownstein et al. 2005; Ogden et al. 2010) and facilitating the spread of $I$. ricinus into higher

* Corresponding author: Zoonoses Division, Centre for Foodborne Environmental and Zoonotics Infectious Diseases, Public Health Agency of Canada, 3200 Sicotte Street, PO 5000, Saint-Hyacinthe, Québec, Canada J2S 7C6. E-mail: jules.konan.koffi@phac-aspc.ca $\dagger$ These authors contributed equally to the work. latitudes and altitudes in Europe (Lindgren et al. 2000; Jaenson and Lindgren, 2011). In the USA, the highest densities of $I$. scapularis occur in the northeastern and north Midwestern states that are close to or border Canadians provinces. In Canada, populations of $I$. scapularis are establishing in the southern parts of central and eastern provinces, thereby accelerating the emergence of LD in those areas (Ogden et al. 2006, 2008, 2014; Bouchard et al. 2015). The expanding geographical distribution of ticks in Canada has been associated with an 8 -fold increase in reported LD incidence (from 128 to 917 cases) from 2009 to 2015 (Ogden et al. 2015; Public Health Agency of Canada, 2016).

Since the early 1990s, a passive tick surveillance system has been operated in Quebec by the Laboratoire de santé publique du Québec (LSPQ), in collaboration with the National Microbiology Laboratory (NML) (Ogden et al. 2006, 2010). The current passive tick surveillance programme is a monitoring system for ticks that are collected from human patients and pets by participating medical and veterinary clinics and submitted to LSPQ for

Parasitology (2017), 144, 877-883. C Cambridge University Press 2017. This is an Open Access article, distributed under the terms of the Creative Commons Attribution-NonCommercial-NoDerivatives licence (http://creativecommons.org/licenses/by-nc-nd/4.0/), which permits non-commercial re-use, distribution, and reproduction in any medium, provided the original work is unaltered and is properly cited. The written permission of Cambridge University Press must be obtained for commercial re-use or in order to create a derivative 
species identification (Ogden et al. 2006). Ticks identified as $I$. scapularis are subsequently shipped to the NML where they are tested for tick-borne pathogens. Data yielded by the surveillance system are compiled annually, forming a database of submitted ticks containing information on tick species, instar, level of engorgement, host species, locality of acquisition and infection prevalence for four tick-borne pathogens. In 2014, 2549 I. scapularis collected in Québec were submitted in the passive surveillance system, representing $55 \%$ of the total number of ticks of all species submitted, and a continuing increase in submissions from the 2182 and 1516 ticks submitted in 2013 and 2012, respectively (Adam-Poupart et al. 2015). The passive surveillance data are used by epidemiologists to determine areas into which populations of $I$. scapularis may be expanding and where, therefore, LD and other TBTs may pose a risk to the population. (Ogden et al. 2010). This knowledge allows public health authorities to target their prevention strategies such as environmental control and messaging to the public and health practitioners to risk areas (Ogden et al. 2014; Bouchard et al. 2015).

This passive tick surveillance system also helps to track the expansion of LD risk in Canada in time and space (Ogden et al. 2006, 2010; Koffi et al. 2012; Leighton et al. 2012). However, the resource needs for this system increase each year as the ticks spread further into Canada. Here we explore the feasibility of an alternative 'citizen science' surveillance method using an electronic platform where digital images of ticks can be submitted for identification, an approach similar to that conducted in several European countries for mosquito surveillance (Kampen et al. 2015). As a first step towards the exploration of this alternative approach for tick surveillance, the present study aimed to: (1) determine if tick identification can be perform based on digital images of ticks submitted through a web platform named 'e'Tick'; and (2) test the capacity of the web platform to manage the online submission of tick images and related collection information. We assessed the usefulness and practicability of the electronic system for identifying ticks and compared its sensitivity and specificity relative to the conventional passive tick surveillance system based on paper copies of collection information and direct examination of tick specimens submitted by veterinary clinics in the province of Quebec, Canada.

\section{MATERIALS AND METHODS}

\section{Data acquisition and sampling period}

The minimum number of tick submissions $(N)$ required to compare the sensitivity and specificity of 'e'Tick' to the conventional system was estimated at 245 using the method of Greiner and Gardner (2000) based on the standard formula for estimating sensitivity and specificity

$$
N=\left(Z_{1-\alpha / 2} / e\right)^{2} \theta(1-\theta),
$$

where $Z_{1-\alpha / 2}$ (the number of standard deviations from the mean of a normal distribution required to contain 0.975 of the area) $=1.96, \alpha=0.05, \boldsymbol{\theta}$ was the assumed $a$ priori estimate of sensitivity or specificity (set at $0 \cdot 8$ ), and $\boldsymbol{e}$ was the error margin on the estimate (set at $\pm 0 \cdot 05$ ) [using recommended values in Greiner and Gardner (2000)].

A pilot test conducted in fall 2014 with ten voluntary veterinary clinics in Quebec to ensure that the web platform was simple and flexible enough yielded ten high-quality submitted digital images. In 2015 , to reach our target of 245 submissions (but anticipating a participation rate of $<40 \%$ based on the 2014 pilot), 122 clinics who had submitted at least five ticks to the passive surveillance programme the previous year were contacted by telephone and e-mail to request their voluntary participation to the project.

Adult I. scapularis are active mostly in spring and autumn but other species are mostly active in summer (Ogden et al. 2010; Bouchard et al. 2013). Therefore, the 2015 sampling season extended from April to December to ensure the collection of multiple tick species.

\section{Electronic platform and data submission procedure}

The 'e'Tick' platform (www.e'Tick.ca) was created and hosted at Bishop's University (BU), Sherbrooke, Quebec. Participating veterinary clinics were provided with instructions for opening a password-protected account, taking good quality pictures of tick specimens, and submitting all relevant data on the electronic platform. Technical assistance (via e-mail messages and telephone) was provided to the users throughout the project. For each submission, users were asked to fill out an online questionnaire about the tick specimen, which contained the same fields as the paper version used in the existing passive surveillance programme (collection date and locality, host species, if the animal had travelled away from the owner's residence during the previous 2 weeks, and if so, where), and to submit up to three digital images of the specimen. Following the completion of data submission on 'e'Tick.ca', a 1 page PDF document containing all collection information was produced (with a copy automatically sent to the user via e-mail) and a unique identification number was attributed to the specimen, allowing for the future cross-referencing of images with the actual specimen. The PDF document was then printed and shipped to the LSPQ, along with the tick placed in a sealed container marked with the 
unique identifier number. All electronic data were stored on a secure server hosted at BU.

\section{Identification procedure}

To prevent bias, image-based and specimen-based identifications of ticks were conducted independently. The digital images were identified by entomologist co-author J. Savage and the corresponding specimens were identified according to the routine passive tick surveillance system at the LSPQ. The identification results from each institution were independently collated by the Public Health Agency of Canada. A summary framework of the data collection and transfer process amongst pet owners, veterinary clinics, the laboratory, the 'eTick' platform and the researchers is shown in Fig. 1.

Due to their small size, ticks can be challenging to photograph, especially when partially or completely engorged with blood. To ensure high-quality pictures where diagnostic features are clearly visible, guidelines on how to take high-quality images of ticks for species identification (including example images) were sent to the veterinary clinics beforehand. The guidelines requested the following key elements: (i) that the specimen was orientated so that key diagnostic features of the head, dorsal shield and ventral surface can be clearly visible; (ii) that the specimen was in focus; and (iii) that an object or ruler would be added in the background for scaling purposes. Both tick images and tick specimens were identified using the taxonomic keys of Clifford et al. (1961) for larvae, and of Keirans et al. (1996) for nymphs, and for adults.

\section{Statistical analyses}

The Kappa statistic with two categories, 'Ixodes scapularis' and 'not Ixodes scapularis', was used to measure the degree of agreement between the two identification methods. Kappa gives a numerical rating of the degree to which agreement is actually present ('observed' agreement) compared to how much agreement would be expected to be present by chance alone ('expected' agreement). It is a measure of how different the observed agreement is from the expected agreement, standardized to a -1 to 1 scale, where 1 is perfect agreement, 0 is exactly what would be expected by chance, and negative values indicate agreement less than chance, i.e. potential systematic disagreement between the observers (Landis and Koch, 1977; Viera and Garrett, 2005; McHugh, 2012). Confidence intervals (CI) for Kappa and $P$ value were calculated. $P$ values were calculated using Fisher's exact test, with the specimen-based identification used as the gold standard.

The sensitivity and specificity of picture-based identification as well as the positive and negative

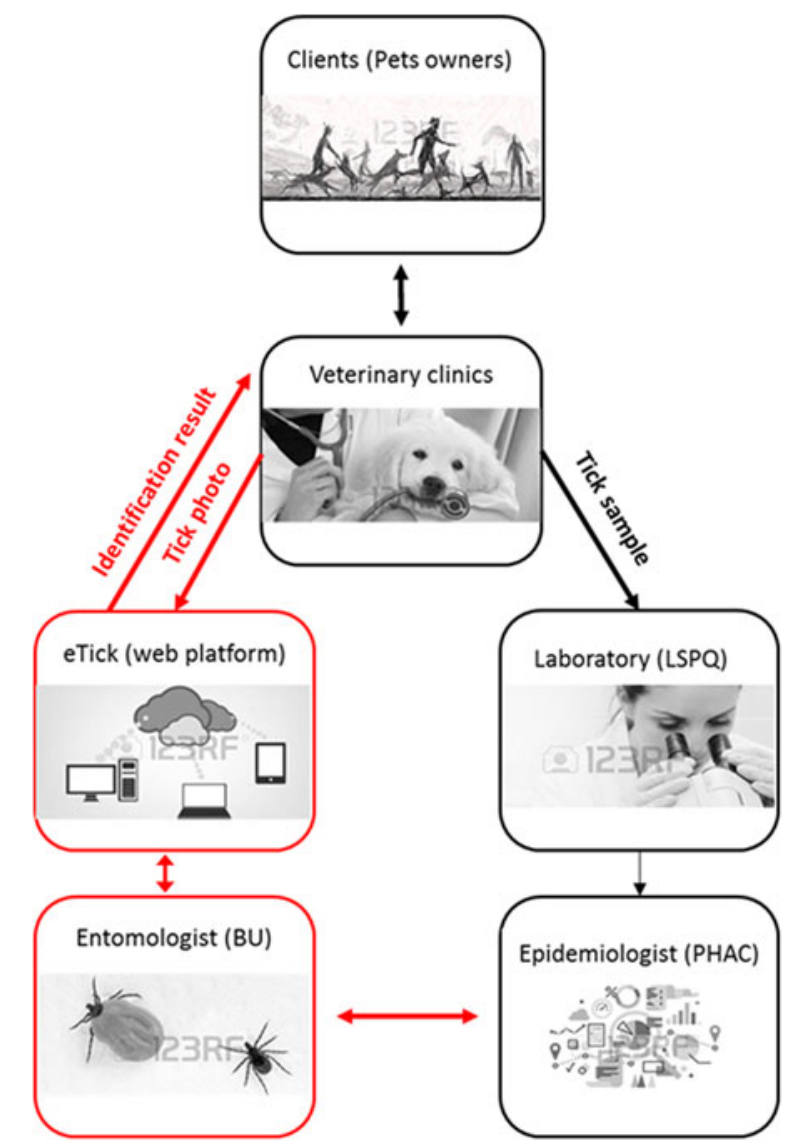

Fig. 1. eTick conceptual framework (preliminary project involving veterinary clinics). LSPQ, Laboratoire de santé publique du Québec (Provincial Public Health Laboratory) PHAC, Public Health Agency of Canada; BU, Bishop's University.

predictive values were also calculated. Sensitivity (Se) and specificity (Sp) were calculated as follows:

$$
\begin{aligned}
\mathrm{Se} & =\mathrm{TP} /(\mathrm{TP}+\mathrm{FN})(\text { where } \mathrm{TP} \\
& =\text { True Positive, } \mathrm{FN}=\text { False Negative }) \\
\mathrm{Sp} & =\mathrm{TN} /(\mathrm{TN}+\mathrm{FP})(\text { where } \mathrm{TN} \\
& =\text { True Negative and } \mathrm{FP}=\text { False Positive }) .
\end{aligned}
$$

All analyses were done using STATA version $11 \cdot 0$ (College Station, TX).

\section{RESULTS}

A total of 455 electronic submissions were sent to eTick.ca during the study. These came from 41 veterinary clinics located in 12 administrative regions of Quebec. Mauricie-et-Centre-du-Québec was the region with the most participating veterinary clinics ' $n=11$ ' and the region from which the greatest number of ticks was submitted ' $n=102$ ', followed by the regions of Lanaudière, Laurentides and Laval with 62, 54 and 48 tick submissions, respectively (Fig. 2). The mean number of tick submissions per clinic was $9 \cdot 31$ (S.D. $=8 \cdot 52)$. Matching 


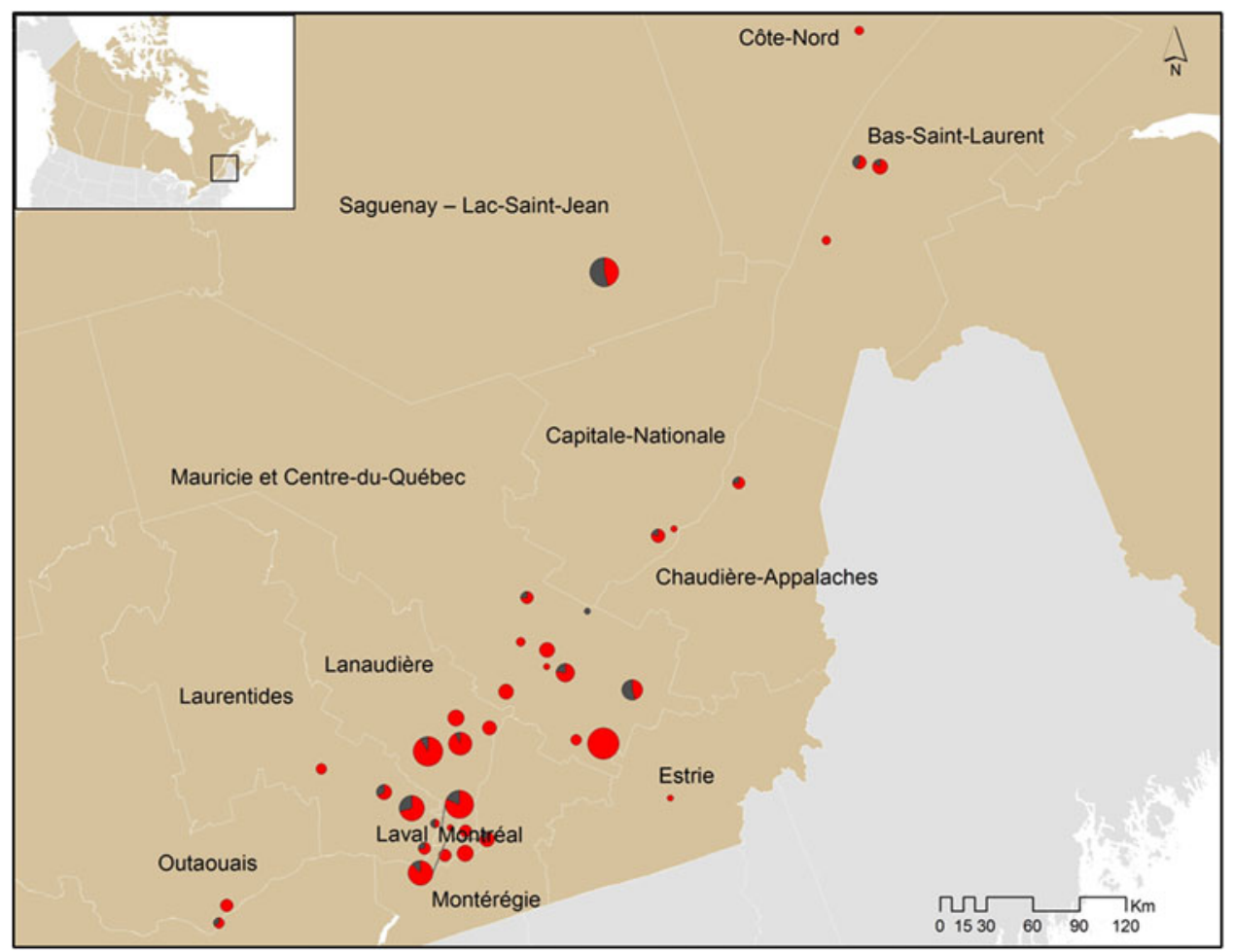

Fig. 2. Proportion and distribution of I. scapularis (red points) and I. cookei (black points) ticks correctly identified in 2014-2015 (i.e. photo and tick specimens) per veterinary clinic in Quebec, Canada. *Dot size varies according to the total number of ticks submitted (see Table 1 for further details).

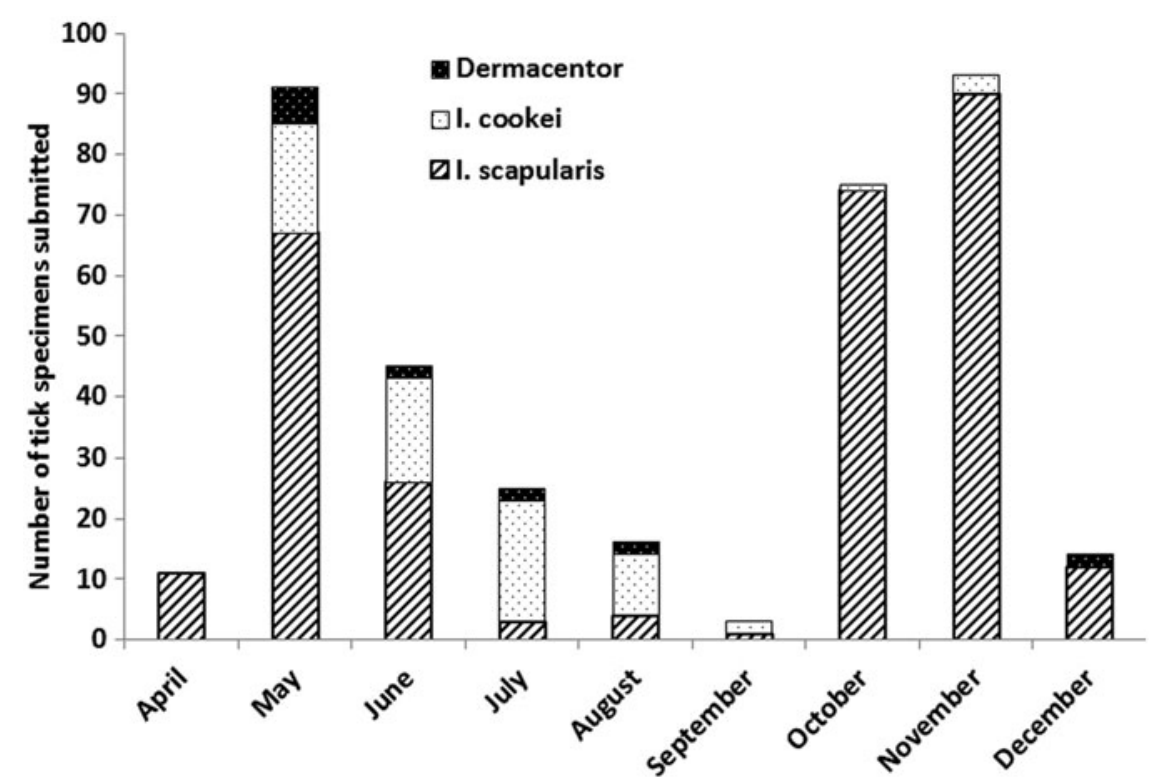

Fig. 3. Seasonal distribution of the main tick species from Quebec, Canada, submitted to eTick.ca in 2014-2015.

tick samples were not transmitted to the laboratory for $16 \%(73 / 455)$ of electronic submissions; therefore, a total of $382(83.96 \%)$ tick records were submitted in both surveillance systems and were available for comparison.

Among the 382 tick records submitted in both surveillance systems, specimen-based identification at the LSPQ yielded seven species; I. scapularis
(294), I. cookei (70), Dermacentor variabilis (11), D. albipictus (3), I. muris (2), Amblyomma americanum (1) and Rhipicephalus sanguineus (1). The two most abundant species, I. scapularis and I. cookei had mostly overlapping distributions: I. scapularis specimens were mostly submitted from Mauricie-etCentre-du-Québec (28.42\%), Lanaudière (19.18\%), Laval $(13 \cdot 01 \%)$ and Laurentides $(11 \cdot 22 \%)$, whereas 
I. cookei was mostly submitted from Laurentides $(25 \cdot 00 \%)$, Mauricie-et-Centre-du-Québec (22.22\%), Saguenay-Lac-Saint-Jean (19.44\%) and Laval (9.72\%) (Fig. 2). Ixodes scapularis showed two abundance peaks, one in the spring and the other one in the fall whereas most $I$. cookei and Dermacentor spp. were recorded in spring and summer (Fig. 3). These seasonal patterns are in accordance with what has been observed in active and passive surveillance in these regions (Ogden et al. 2006; Bouchard et al. 2011).

For the 382 records submitted online with matching specimen-based identification from the LSPQ, $8.90 \%$ of images were classified as high quality, $46 \cdot 07 \%$ as good, $19 \cdot 11 \%$ as poor and $25 \cdot 92 \%$ as unusable. Unusable images (98) were excluded from further analyses; therefore 284 (74.34\%) submissions were of suitable quality to allow identification to the species level for Ixodes spp. ticks and to the generic level for Dermacentor spp. This number (284), provided sufficient statistical power to estimate agreement, sensitivity and specificity of the two methods of identification.

Of these 284 submissions of sufficient quality for image-based identification, 276 were identified correctly, therefore reaching an agreement of $97 \cdot 18 \%$ with specimen-based identification (Table 1). Eight ticks, all adult females, were not correctly classified by examination of digital images: three $I$. scapularis were classified as I. cookei, three I. cookei were classified as I. scapularis, and two I. muris were classified as I. scapularis. The Kappa statistic of inter-rater agreement $(218 / 5$ and $3 / 58)$ was 0.92 (95\% confidence interval $=$ ' $[0 \cdot 86-0 \cdot 97]$ ' and was significantly different from $0(Z=15 \cdot 46, P<0 \cdot 001)$. 'The sensitivity and specificity of the image-based identification of I. scapularis was 98.64 and $92.06 \%$ respectively with a predicted positive value of $97 \cdot 76 \%$ and a predicted negative value of $95 \cdot 08 \%$.'

Of the 284 records having good quality enough to allow image-based identification, 252 specimens were on good shape to determine the state of engorgement; 68 were engorged, 174 were partially engorged and 12 were non-engorged. Overall the identification output were similar for the engorged and partially engorged ticks (97.06 vs $97 \cdot 13 \%)$ and higher for the non-engorged ticks $(100 \%)$ (Table 2). However, this observed difference is not statistically significant (Fisher exact test $=1 \cdot 00$ ). The status of engorgement does not seem to influence the ability of entomologists to perform the image-based identification. There was only nine specimens of Dermacentor ticks for which the state of engorgement was available and there was no Dermacentor tick non-engorged recorded in the study (Table 2). The small number of Dermacentor ticks (' $n=9$ ') in this study does not allow a fairly comparison of the precision of identification between Dermacentor and Ixodes species.

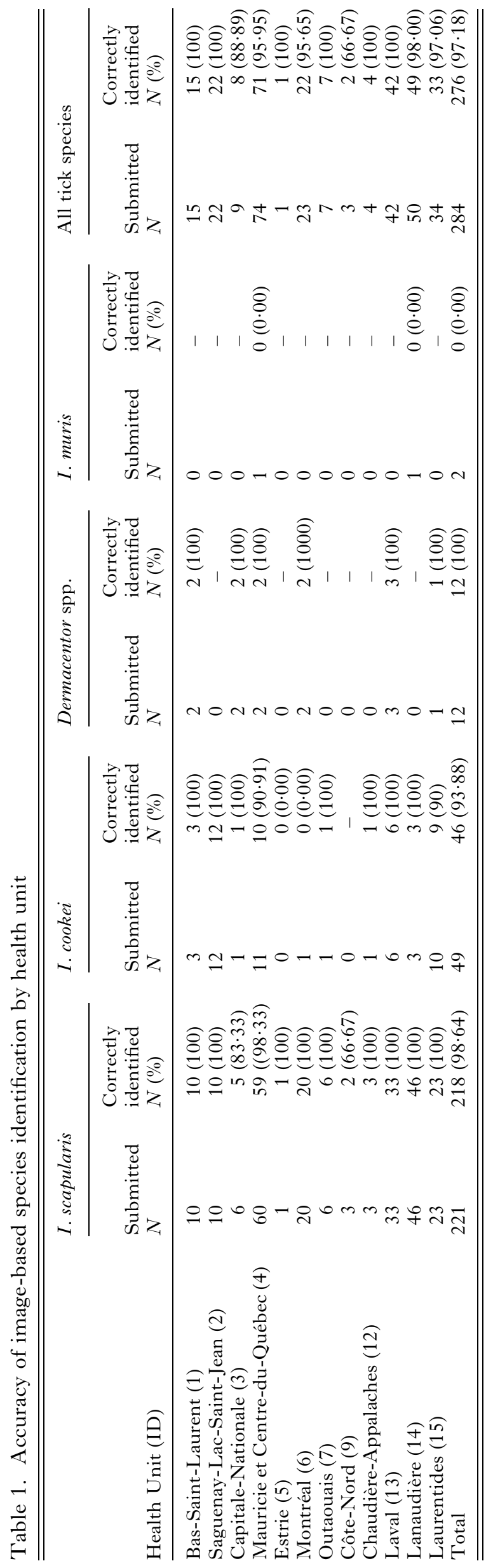


Table 2. Accuracy of image-based species identification by state of engorgement

\begin{tabular}{|c|c|c|c|c|c|c|c|}
\hline \multirow[b]{3}{*}{ Species } & \multirow[b]{3}{*}{$\begin{array}{l}\text { Ticks } \\
\text { identified } N\end{array}$} & \multicolumn{6}{|c|}{ State of engorgement } \\
\hline & & \multicolumn{2}{|c|}{ Engorged } & \multicolumn{2}{|c|}{ Partially engorged } & \multicolumn{2}{|c|}{ Non-engorged } \\
\hline & & $N$ & $\begin{array}{l}N(\%) \text { correctly } \\
\text { identified }\end{array}$ & $N$ & $\begin{array}{l}N(\%) \text { correctly } \\
\text { identified }\end{array}$ & $N$ & $\begin{array}{l}N(\%) \text { correctly } \\
\text { identified }\end{array}$ \\
\hline I. scapularis & 206 & 53 & $52(98 \cdot 11)$ & 150 & $148(98 \cdot 67)$ & 3 & $3(100)$ \\
\hline I. cookei & 37 & 12 & $11(91 \cdot 67)$ & 16 & $15(93 \cdot 75)$ & 9 & $9(100)$ \\
\hline I. muris & 2 & 0 & - & 2 & $0(0 \cdot 00)$ & 0 & - \\
\hline Dermacentor & 9 & 3 & $3(100)$ & 6 & $6(100)$ & 0 & - \\
\hline Total & 254 & 68 & $66(97 \cdot 06)$ & 174 & $169(97 \cdot 13)$ & 12 & $12(100)$ \\
\hline
\end{tabular}

\section{DISCUSSION}

Ixodes scapularis populations are spreading northwards into eastern and central Canada and in different parts of the northern hemisphere. Associated with the expanding tick populations, TB'Ts such as LD, babesiosis, anaplasmosis, Powassan virus infection and Borrelia miyamotoi infection are emerging and threatening the health of populations living in these areas and their pets (Lindgren et al. 2000; Ogden et al. 2006; Bacon et al. 2008; Leighton et al. 2012; Bouchard et al. 2015). Knowing where Lyme risk is occurring guides the targeting of public health interventions such as environmental control and messaging to the public and medical and veterinary practitioners to facilitate prevention and early diagnosis of tickborne infections in people (Ogden et al. 2010) and domesticated animals (Bouchard et al. 2015). Citizen-based surveillance methods can aid in detecting emerging tick populations in a timely fashion (Madder et al. 2012), and can complement existing passive and active surveillance programmes.

Our results showed that images taken of ticks by animal health care professionals (veterinarians and/ or animal health technicians) were of a quality suitable to assess the tick species in almost three quarters of cases. This suggests that digital images of ticks can be used for identification. Our study yielded an agreement of $97 \cdot 18 \%$ and a Kappa statistic of 0.92 between identification of ticks by digital images and examination of the actual specimen. This result demonstrates that picture-based identification of ticks, when accompanied by georeferenced collection data, could complement the existing surveillance systems to identify localities where the vectors of LD are spreading, and where there is a potential risk for the public from LD and emerging TBTs. The study shows that the state of engorgement does not have an impact on the result of identification. Even if the tick is engorged, factors such as the scutum, the location were the tick has been collected, and time of year can guide to image-based identification. Further study with a larger sample size is needed to better analyse the impact of different factors on the quality of image and image-based identification and to compare the precision of identification between tick species.

While the objective of this system is to be able to detect I. scapularis ticks, it appears capable of identifying other tick species that may be of public health significance. These include $I$. cookei (the groundhog tick) which transmits Powassan virus (Hinten et al. 2008) and Dermacentor spp., which may be the vector of spotted fever bacteria (Openshaw et al. 2010). Some of these tick vector species and the pathogens that can be associated with them may pose a threat to the health of populations living in areas where population of ticks are established and identifying their geographic occurrence is also of public health importance.

The internet, with its widespread use and easy accessibility, offers great possibilities to help overcome some of the challenges of traditional surveillance systems. If available to the public, an electronic image-based tick surveillance tool could speed up the monitoring of tick occurrence and activity by removing the need for postage of tick specimens, help reduce the cost of surveillance; and allow rapid feedback to the submitter on both the type of tick they collected and more general information on how to prevent TB'Ts and on tick bite management.

The methodology used in this study appeared to work well in general; however, the approach has some limitations. First, several clinics could not participate in the study (or their images were rejected) because their photographic equipment produced images that were not usable for tick identification. This was especially common with clinics using tablets (data not shown), and variation amongst devices in the quality of digital images used for the purposes described here requires further study. In addition, tick specimens that were severely damaged (e.g. decapitated) or badly desiccated could not be identified based on the digital images. Lastly, ticks identified based only upon digital images obviously would not be available for pathogen testing and as a result, additional surveillance (and collection of actual tick specimens) would 
need to be conducted in order to document the prevalence of tick-associated pathogens. A rigorous application of guidelines on how to take highquality images of ticks for species identification could allow avoiding some of these limitations.

In conclusion, this study suggests that ticks (at least those most common in Quebec) can be identified using digital images. Based on our experience with the electronic platform and our interactions with veterinary staff, we also conclude that data submission and management through the 'eTick' electronic platform worked well for both users and data managers. Consequently, our next step will be a performance assessment of the electronic platform when adapted for use by the general public. Such a web-based public system could not only become a useful and relatively inexpensive complement to the existing surveillance programme for monitoring tick populations, it could also be used to rapidly disseminate information about tick abundance hot-spots, tickbite prevention and TBTs to concerned citizens.

\section{ACKNOWLEDGEMENTS}

We would like to thank Xinbao Zhang (programmer) for the implementation of the 'eTick' website and for providing technical support to the team throughout this phase of the project. We also thank Elisabeth Allard (Bishop's University) for her input into data management and Maxim Larrivée (Insectarium de Montréal) for providing advice based on his experience as the founder and director of e-butterfly.org. Finally, this work would not have been possible without the LSPQ technical staff, Annie Rouleau, Alexandra Cecan and Marie Lemieux for their expertise in tick identification.

\section{FINANCIAL SUPPORT}

This study was supported by the Public Health Agency of Canada (electronic platform implementation), the LSPQ (tick specimen identification) and by Bishop's University (website hosting and maintenance, image-based identification of tick species and data management).

\section{REFERENCES}

Adam-Poupart, A., Thivierge, K., Ouhoummane, N., Irace-Cima, A. and Milord, F. (2015). Surveillance de la maladie de Lyme et des autres maladies transmises par la tique Ixodes scapularis au Québec: année 2014. Gouvernement du Québec, Québec, Canada.

Bacon, R. M., Kugeler, K. J. and Mead, P. S. (2008). Surveillance for Lyme Disease - United States, 1992-2006. MMWR Surveill Summ. 57, $1-9$

Bouchard, C., Beauchamp, G., Nguon, S., Trudel, L., Milord, F., Lindsay, L. R., Belanger, D. and Ogden, N. H. (2011). Associations between Ixodes scapularis ticks and small mammal hosts in a newly endemic zone in southeastern Canada: implications for Borrelia burgdorferi transmission. Ticks and Tick-Borne Diseases 2, 183-190.

Bouchard, C., Beauchamp, G., Leighton, P. A., Lindsay, R., Belanger, D. and Ogden, N. H. (2013). Does high biodiversity reduce the risk of Lyme disease invasion? Parasites $\mathscr{F}^{\circ}$ Vectors 6, 195

Bouchard, C., Leonard, E., Koffi, J. K., Pelcat, Y., Peregrine, A., Chilton, N., Rochon, K., Lysyk, T., Lindsay, L. R. and Ogden, N. H. (2015). The increasing risk of Lyme disease in Canada. Canadian Veterinary fournal 56, 693-699.

Brownstein, J. S., Holford, T. R. and Fish, D. (2005). Effect of climate change on Lyme disease risk in North America. Ecohealth 2, 38-46.
Clifford, C. M., Anastos, G. and Elbl, A. (1961). The larval ixodid ticks of the eastern United States. Miscellaneous Publications of the Entomological Society of America 2, 215-244.

Greiner, M. and Gardner, I. A. (2000). Epidemiologic issues in the validation of veterinary diagnostic tests. Preventive Veterinary Medicine 45, 3-22.

Hinten, S. R., Beckett, G. A., Gensheimer, K.F., Pritchard, E., Courtney, T.M., Sears, S.D., Woytowicz, J.M., Preston, D. G., Smith, R. P., Jr., Rand, P.W., Lacombe, E. H., Holman, M. S., Lubelczyk, C. B., Kelso, P.T., Beelen, A.P., Stobierski, M. G., Sotir, M. J., Wong, S., Ebel, G., Kosoy, O., Piesman, J., Campbell, G. L. and Marfin, A. A. (2008). Increased recognition of Powassan encephalitis in the United States, 1999-2005. Vector-Borne and Zoonotic Diseases 8, 733-740. Jaenson, T. G. and Lindgren, E. (2011). The range of Ixodes ricinus and the risk of contracting Lyme borreliosis will increase northwards when the vegetation period becomes longer 1. Ticks Tick Borne Dis. 2, 44-49.

Kampen, H., Medlock, J. M., Vaux, A. G., Koenraadt, C. J., van Vliet, A. J., Bartumeus, F., Oltra, A., Sousa, C. A., Chouin, S. and Werner, D. (2015). Approaches to passive mosquito surveillance in the EU 8. Parasites \& Vectors $8,9$.

Keirans, J.E., Hutcheson, H. J., Durden, L. A. and Klompen, J. S. (1996). Ixodes (Ixodes) scapularis (Acari:Ixodidae): redescription of all active stages, distribution, hosts, geographical variation, and medical and veterinary importance. Fournal of Medical Entomology 33, 297-318.

Koffi, K. J., Leighton, P. A., Pelcat, Y., Trudel, L., Lindsay, L. R., Milord, F. and Ogden, N. H. (2012). Passive Surveillance for I. scapularis ticks: enhanced analysis for early detection of emerging Lyme disease risk. Fournal of Medical Entomology 49, 400-409.

Landis, J. R. and Koch, G. G. (1977). The measurement of observer agreement for categorical data 3. Biometrics 33, 159-174.

Leighton, P. A., Koffi, K. J., Pelcat, Y., Lindsay, L. R. and Ogden, N. H., British Ecological Society (2012). Predicting the speed of tick invasion: an empirical model of range expansion for the Lyme disease vector Ixodes scapularis in Canada. Fournal of Applied Ecology 49, 457-464.

Lindgren, E., Talleklint, L. and Polfeldt, T. (2000). Impact of climatic change on the northern latitude limit and population density of the diseasetransmitting European tick Ixodes ricinus. Environmental Health Perspectives 108, 119-123.

Madder, M., Walker, J. G., Van Rooyen, J., Knobel, D., Vandamme, E., Berkvens, D., Vanwambeke, S. O. and De Clercq, E. M. (2012). e-Surveillance in animal health: use and evaluation of mobile tools. Parasitology 139, 1831-1842.

McHugh, M. L. (2012). Interrater reliability: the kappa statistic 1. Biochemia Medica (Zagreb.) 22, 276-282.

Ogden, N. H., Trudel, L., Artsob, H., Barker, I. K., Beauchamp, G., Charron, D., Drebot, M. A., Galloway, T.D., O'Handley, R. and Thompson, R. A. (2006). Ixodes scapularis ticks collected by passive surveillance in Canada: analysis of geographic distribution and infection with the Lyme borreliosis agent Borrelia burgdorferi. Fournal of Medical Entomology 43, 600-609.

Ogden, N.H., St-Onge, L., Barker, I. K., Brazeau, S., BigrasPoulin, M., Charron, D. F., Heagy, A., Lindsay, L. R., Maarouf, A., Michel, P., Milord, F., O'Callaghan, C. J., Trudel, L. and Thompson, R. A. (2008). Risk maps for range expansion of the Lyme disease vector, Ixodes scapularis, in Canada now and with climate change. International Fournal of Health Geographics 7, 24.

Ogden, N. H., Bouchard, C., Kurtenbach, K., Margos, G., Lindsay, L. R., Trudel, L., Nguon, S. and Milord, F. (2010). Active and passive surveillance and phylogenetic analysis of Borrelia burgdorferi elucidate the process of Lyme disease risk emergence in Canada. Environmental Health Perspectives 118, 909-914.

Ogden, N.H., Koffi, J., Pelcat, Y. and Lindsay, L. R. (2014). Environmental risk from Lyme disease in central and eastern Canada: a summary of recent surveillance information. Canada Communicable Disease Report 40, 74-82.

Ogden, N.H., Koffi, K. J., Leonard, E., Fleming, S., Monbourquette, D. C., Sanford, C., Raafat, G. R., Jain-Sheehan, N., Moore, S., Russel, C., Baydack, R., Granstrom, M., Lachance, L., Simmonds, K. and Scott, A. N. (2015). Surveillance for Lyme disease in Canada, 2009-2012. Canada Communicable Disease Report 41, 132-145. Openshaw, J. J., Swerdlow, D. L., Krebs, J.W., Holman, R.C., Mande1, E., Harvey, A., Haberling, D., Massung, R.F. and McQuiston, J.H. (2010). Rocky mountain spotted fever in the United States, 2000-2007: interpreting contemporary increases in incidence. American Fournal of Tropical Medicine and Hygiene 83, 174-182.

Public Health Agency of Canada (2016). Surveillance for Lyme Disease in Canada. Government of Canada, Ottawa, Canada.

Viera, A. J. and Garrett, J. M. (2005). Understanding interobserver agreement: the kappa statistic 1. Family Medicine 37, 360-363. 